\title{
Human embryonic derived neural progenitor cells improves neurological scores following brain ischemia/ reperfusion: Modulation of blood and brain tissue MicroRNA-210
}

\author{
Leila Arab', Aslan Fanni², Shiva Nemati², Ehsan Arefian³, Jafar Ai" ${ }^{* 4}$, Tahmineh Mokhtari 5,6, \\ Maryam Farahmandfar ${ }^{7}$, Nasser Aghdami ${ }^{2}$, Gholamreza Hassanzadeh ${ }^{1,8,9}$
}

\author{
'Department of Neuroscience and Addiction Studies, School of Advanced Technologies in Medicine, Tehran University of Medical Sciences, Tehran, Iran. \\ ${ }^{2}$ Department of Stem cells and Developmental Biology, Cell Science Research Center, Royan Institute for Stem Cell Biology and Technology, ACECR, Tehran, Iran. \\ ${ }^{3}$ Department of Microbiology, School of Biology College of Science, University of Tehran, Tehran, Iran. \\ ${ }^{4}$ Department of Tissue Engineering and Applied Cell Sciences, School of Advanced Technologies in Medicine, Tehran University of Medical Sciences, Tehran, Iran. \\ ${ }^{5}$ CAS Key Laboratory of Mental Health, Institute of Psychology, Beijing, China; \\ ${ }^{6}$ Department of Psychology, University of Chinese Academy of Sciences, Beijing, China \\ 'Department of Neuroscience and Addiction Studies, School of Advanced Technologies in Medicine, Electrophysiology Research Center, Neuroscience Institute, \\ Tehran University of Medical Sciences, Tehran, Iran. \\ ${ }^{8}$ Legal Medicine Research Center, Legal Medicine Organization, Tehran, Iran. \\ ${ }^{9}$ Department of Anatomy, School of Medicine, Tehran University of Medical Science, Tehran, Iran. \\ \#2 co-second author \#3 co-third author \\ Correspondence to: Gholamreza Hassanzadeh (hassanzadeh@tums.ac.ir )
}

(Submitted: 13 March 2020 - Revised version received: 28 March 2020 - Accepted: 11 May 2020 - Published online: 26 June 2020)

\begin{abstract}
Objective In this study, we evaluated the effects of human embryonic derived neural progenitor cells on neurological score, histopathological changes, and miRNA-210 as biomarkers of regeneration.

Methods The animals were randomly divided into the four groups: Sh (sham), MCAO (middle cerebral artery occlusion), MCAO+PBS, and MCAO+Cell. One day after MCAO induction, embryonic derived neural progenitor cells (hESC-NPCSGFP) or PBS were injected intracerebroventriculary in $\mathrm{MCAO}+\mathrm{Cell}$ or $\mathrm{MCAO}+\mathrm{PBS}$ groups. On day 1, 2, 3, and 7 after ischemia induction, the neurological score was tested in each rat. At $48 \mathrm{~h}$, the expression of miRNA-210 was evaluated and 7 days after, the pathological assessments were performed by H\&E staining

Results Neurological score showed the promotion of functional recovery in MCAO+Cell group. Based on H\&E staining, the percentage of neural death in ischemic region reduced in $\mathrm{MCAO}+$ Cell group. The miRNA-210 significantly upregulated in both brain and blood samples. Conclusion According to the findings, hESC-NPCS GPP injection could upregulate the miRNA-210 of tissue and blood to support the neuroprotective and regenerative effect of $\mathrm{hESC}-\mathrm{NPCS}{ }^{\mathrm{GPP}}$ in the ischemic lesion and improved the neurological score and reduce the neural death in ischemic region.
\end{abstract}

Keywords Embryonic stem cell; Neural death; Micro-RNA-210; Brain ischemia; Rat

\section{Introduction}

Stroke is the most prevalent vascular accident of central nervous system among middle aged individuals. The sensorymotor dysfunction, cognitive impairments, and declined quality of life are the side effects of stroke in suffered patients. ${ }^{1}$ Unfortunately, no effective pharmaceutical treatment has been introduced, only endovascular approaches or rehabilitation may reduce the severity of symptoms. ${ }^{2}$ In the field of regenerative medicine, there are numerous preclinical and clinical studies that demonstrated the therapeutic effect of stem cell transplantation in various neurodegenerative diseases such as ischemic stroke. ${ }^{3-5}$ Embryonic stem cells (ESC), the pluripotent stem cells, can differentiate to different lineages. So that, under defined protocol, embryonic stem cells has been shown to differentiate to neural progenitor cells (NPC). ${ }^{6}$ After transplantation of embryonic derived neural progenitor cells (ES-NPCs) in model of middle cerebral artery occlusion (MCAO) in the rats, the cells are capable to migrate toward ischemic site, proliferate and differentiate to the neurons and glial cells, and replace the dead cells. ${ }^{7}$ On the other hand, they induce angiogenesis and neurogenesis, decrease the neuroinflammation, and preserve the integrity of blood-brain barrier through bystander effects. ${ }^{8}$ Following the ES-NPCs injection, the size of ischemic area reduced and the sensory-motor function improved. The histological investigation also revealed positive findings in favor of neural tissue repair. ${ }^{6,9,10}$

Moreover, different types of biomarkers such as microRNAs (miRNAs) appear in blood and brain tissue following ischemia. The miRNAs are small non-coding and single-stranded RNAs that regulate many internal processes such as cell proliferation, differentiation, development, cell cycle, apoptosis, etc. In addition to tissues, they are present in serum or plasma in the form of complexes and macrovesicles. ${ }^{11}$ The previous studies exposed that a wide spectrum of miRNAs identified, in the blood and brain tissue after MCAO in rats by microarray with both up- and downregulated manner. Clinical studies also confirmed these findings in patients with ischemic stroke. Therefore, the miRNAs are considered as a promising biomarker for prognosis of stroke patients. ${ }^{12-16}$

miRNA-210 has been shown to prevent neuronal apoptosis and with neuroprotective role by suppressing the caspase pathway, induce a balance between bcl-2 and bax expression. ${ }^{17}$ In ischemic condition, mir- 210 plays role as a proangiogenic factor and involves in cell-cycle regulation, DNA damage reconstruction, and neural tissue restoration. ${ }^{17-19}$ 
Also, miRNA-210 improves the stem cell survival via regulation of apoptosis-related protein (caspase 8 associated protein 2). ${ }^{20,21}$ Also, overexpression of Mir-210 induces angiogenesis and neurogenesis in ischemic tissues to compensate decrease hypoperfusion. $^{22}$

We designed this study to evaluate the effect of intracerebroventricul injection (ICVI) of ES-NPCs on fold changes of miRNA-210 in ischemic brain tissue and compared with MCAO and MCAO+Placebo groups, $24 \mathrm{~h}$, and $48 \mathrm{~h}$ after ischemia.

\section{Method and Material}

\section{Animals}

The male Wistar albino rats (260-300 g, 12-week-old) were enrolled into the study, purchased from laboratory animal department of Royan Institute. They were kept in animal room with temperature of $18-24^{\circ} \mathrm{C}, 40--70 \%$ humidity, $12 \mathrm{~h}$ light-12h dark cycle and free access to food and water. They were treated according to the guidelines of Iranian council for use and care of animals and approved by Ethical Committee of Tehran University of Medical Sciences. The rats were randomly divided into four groups:

1. Sham group (Sh): Operated rats without any vascular occlusion which underwent stereotaxic intervention and with experience of ICVI injection ( $\mathrm{n}=8$ rats).

2. MCAO: Rats only with MCAO for $60 \mathrm{~min}(\mathrm{n}=8$ rats).

3. MCAO + PBS (phosphate buffered saline) group: Rats with MCAO for $60 \mathrm{~min}$ which followed by ICVI injection of PBS $(5 \mu \mathrm{l})$ ( $\mathrm{n}=8$ rats).

4. $\mathrm{MCAO}+$ Cell group: Rats with $\mathrm{MCAO}$ for $60 \mathrm{~min}$ which was followed by ICVI injection of cell suspension. $\left(10^{5}\right.$ cells in $5 \mu \mathrm{PBS})$ ( $\mathrm{n}=8$ rats).

\section{Model Induction}

Before MCAO induction, each rat was held in the induction chamber with vaporization of $5 \%$ isoflurane. Then, it was put immediately in supine position on heating pad and heating light with nosecone mask to inhalation of $1-2 \%$ isoflurane during surgery. With a middle neck incision and dissection of the neck soft tissues and muscles, we accessed to the common carotid artery (CCA). In next step, the proximal of CCA and external carotid artery (ECA) were ligated, and an intraluminal 4-0 nylon monofilament (Doccol Co., USA) was inserted into the MCA to occlude its origin under monitoring of blood flow by Laser Doppler flowmeter (Moor Instruments). After $60 \mathrm{~min}$, the filament was removed and the neck incision was sutured..$^{23}$ The body temperature was monitored with rectal temperature probe to remain at $37^{\circ} \mathrm{C} .^{24}$

\section{Cell Preparation and Generating of hESC-NPCs ${ }^{G F P}$}

The hESC (RH6) was received from Royan cell bank, then, their differentiation procedure toward hNPCs has been done according to a standard procedure. ${ }^{25}$ The characteristics of hESC-NPCs ${ }^{\mathrm{GFP}}$ were evaluated using immunofluorescence staining.

\section{Immunofluorescence Staining}

To perform immunofluorescence staining, hNPC-GFP were fixed using 4\% paraformaldehyde (Mallinckrodt, Phillipsburg, NJ), and permeabilized with $0.1 \%$ Triton X-100 (Sigma) for $15 \mathrm{~min}$ at ambient temperature. The cells were incubated with primary antibody for $1 \mathrm{~h}$ at room temperature (RT), washed, and incubated with fluorescein isothiocyanate-conjugated secondary antibodies, antimouse immunoglobulin $\mathrm{M}(\mathrm{IgM})$ (1:100), antimouse $\operatorname{IgG}(1: 200)$, and antirat $\operatorname{IgM}(1: 200)$, as appropriate, for $1 \mathrm{~h}$ at RT. Primary antibodies were Nestin (1:100), SOX1(1:100), GFAP (1:400) to confirm the undifferentiated stage. The cells were analyzed with a fluorescent microscope (Olympus).

\section{ICV Injection}

To perform the ICV injection, after $24 \mathrm{~h}$ the rats were anesthetized with Isoflurane $5 \%$ for induction and $2 \%$ for maintenance), then fixed in stereotaxic frame. The ES-NPCs $\left(1 \times 10^{5}\right.$ cells in $5 \mu \mathrm{PBS}$ ) or PBS was injected with using Hamilton syringe into the right cerebral ventricle at: bregma: $\mathrm{AP}=-0.12$ $\mathrm{mm}, \mathrm{ML}=1.6 \mathrm{~mm}$, and $\mathrm{DV}=4.3 \mathrm{~mm}$.

\section{Modified Neurological Severity Score (mNSS)}

To assess the sensory, motor, reflexes, and balance of rats after MCAO, we use mNSS test, ${ }^{26}$ while the worst score is 0 and the best one is 18 . The test was performed for each rat on day 1,2 , 3 , and 7 after ischemia induction.

\section{miRNA Real-Time Quantitative PCR}

The miRNA expression was measured in the ischemic area and blood samples $48 \mathrm{~h}$ after MCAO. The rats were anesthetized with isoflurane $5 \%$. The cardiac blood samples were taken and the ischemic area of the right hemisphere was isolated and stored in $-80^{\circ} \mathrm{C}$ freezer. Total RNA (plus miRNA) was extracted from brain samples. Single-strand cDNA was synthesized using universal cDNA synthesis kit (Exiqon, Vedbaek, Denmark). Quantification of miRNA-210 was performed with stem-loop real-time PCR. qPCR was performed in triplicate in three separate experiments on an Applied Biosystems Step One Plus real-time PCR machine. The relative expression of miRNA was normalized to the endogenous control U6 expression using the comparative cycle threshold (CT) method.

\section{Hematoxylin and Eosin Staining}

For light microscopy study, the rats were anesthetized with ketamine/xylazine (RaziCo, Iran), and perfused by $0.9 \%$ saline and $4 \%$ paraformaldehyde (PFA, sigma), respectively. The brains were dissected and cut into the sections with 3-5 mm thickness. Then, the sections were post-fixed in 10\% formalin $72 \mathrm{~h}$ at $4^{\circ} \mathrm{C}$. In order to light microscopy analysis, the samples were embedded in paraffin and $5 \mu \mathrm{m}$ coronal sections were prepared by using a rotary microtome (Leica Biosystems, Milan, Italy). One section from each five section was selected and the tissue sections stained with Hematoxylin and eosin (H\&E). Afterward, graded alcohols (70, 80, 90, and 100\% [2 times]) was used to dehydrate the sections. Finally, they were mounted in Canada balsam and prepared for analysis. Study and survey of sections was performed by using a light field microscope (Olympus, CX31, Tokyo, Japan). In cortex field, the intact and ischemic cells considered as dark neurons, were counted in the $\times 400$ images by using a connected camera to the microscope. ${ }^{27}$

\section{Statistical Analysis}

Data analysis was performed with standard statistical software GraphPad Prism, version 6 (GraphPad, La Jolla, CA). One-way 
ANOVA followed by Bonferroni's post-hoc comparisons tests were performed in all statistical analyses. To test the feasibility, we built statistical model by regression analysis. Correlations were estimated by Pearson correlation test. Differences were considered significant at $p<0.05$.

\section{Result}

\section{Characterization of hESC-NPCs ${ }^{\text {GFP }}$}

Generated hNPC (Fig. 1) was evaluated for expression of neural progenitor markers by immunofluorescence staining. The phase contrast microscopy photograph of normal hNPC was shown in Fig. 1a. The hNPC population had highly expressed NESTIN (Fig. 1b), SOX1 (Fig. 1d) with the lower expression of GFAP (Fig. 1c) at their progenitor stage.

\section{Effects of ICV Injection of hESC-NPCs ${ }^{\text {GFP }}$ on the Modified Neurological Severity Score (mNSS) Following I/R Injury}

The results of behavioral functional test (mNSS) showed that the neurological function outcomes significantly improved in MCAO+Cell during a week after injection $(3.71 \pm 0.76 / 18$, $p$-value $<0.001) \quad$ compared with MCAO $(8.29 \pm 1.11$, $p$-value $<0.059)$ and $\mathrm{MCAO}+\mathrm{PBS}(7.71 \pm 1.11, p$-value: 0.230$)$ groups on day 7 (Table 1).

\section{Effects of ICV Injection of hESC-NPCs ${ }^{\text {GFP }}$ on the Neural Cells Death of Ischemic Area Following I/R Injury}

Evaluation of apoptosis by H\&E staining showed that the count of neural cell death (shrunken cells) in MCAO+Cell group is much lesser than ( 50\%) MCAO+PBS group (80\%) and MCAO group ( $80 \%$ ) (Fig. 2 ).

\section{Effects of ICV Injection of $h E S C-N P C s^{\text {GFP }}$ on miRNA-210 Profile (RT-PCR) of Blood and Brain Samples of Ischemic Area Following I/R Injury}

Brain and blood samples from rats in different groups were screened for a total of 72 Rattus norvegicus. The miRNA-210 was found to be present in both the blood and brain $48 \mathrm{~h}$ after reperfusion. Then, the correlation between MCAO-Blood/ Tissue- $48 \mathrm{H}$ was examined (Fig. 3).
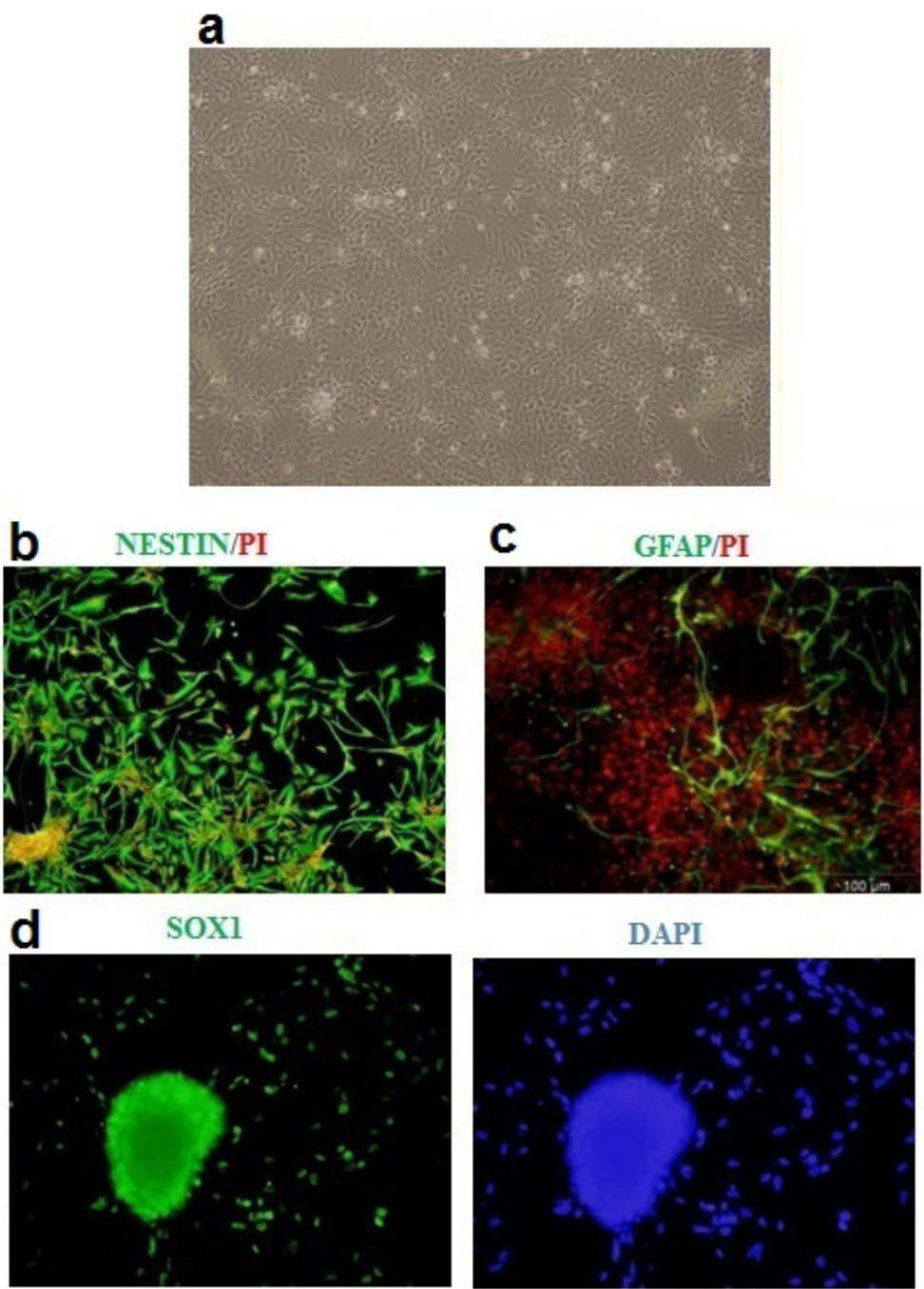

Fig. 1 Characterization of hESC-NPC ${ }^{\text {GFP }}$. (a) Phase contrast microscopy of normal hNPC after generation of RH6. (b-d) Immunofluorescence staining for neural progenitor markers (NESTIN, SOX1, and GFAP). 
Table 1. Effects of ICV injection of hESC-NPCs ${ }^{\text {GFP }}$ on the Modified Neurological Severity Score (mNSS) following I/R injury.

\begin{tabular}{lllllll}
\hline & \multicolumn{5}{c}{ Time (Day) } & \\
\cline { 2 - 5 } Group & Day 1 & Day 2 & Day 3 & Day 7 & p-Value & Pairwise comparisons \\
\hline MCAO (M) & $10.14(1.86)$ & $9.43(1.27)$ & $9.29(0.95)$ & $8.29(1.11)$ & 0.059 & - \\
MCAO+ PBC (P) & $9.43(1.81)$ & $8.57(2.23)$ & $7.29(3.09)$ & $7.71(1.11)$ & 0.230 & - \\
MCAO+ Cell (C) & $10.14(2.27)$ & $7.57(1.40)$ & $6.29(1.38)$ & $3.71(0.76)$ & $<0.001$ & $1>3 ; 1>7 ; 2>7$ \\
P-Value & 0.744 & 0.164 & 0.044 & $<0.001$ & & \\
Pairwise Comparisons & - & - & $C<M$ & $C<M ; C<P$ & & \\
\hline
\end{tabular}

PBS: Phosphate Buffer Saline.

Values are given as mean (SD).

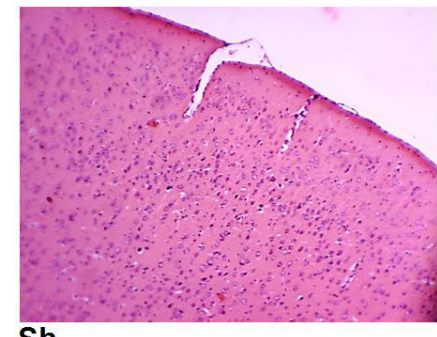

Sh

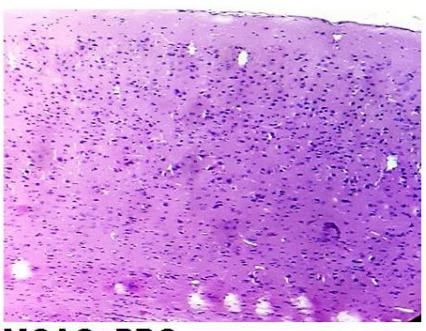

MCAO+PBC

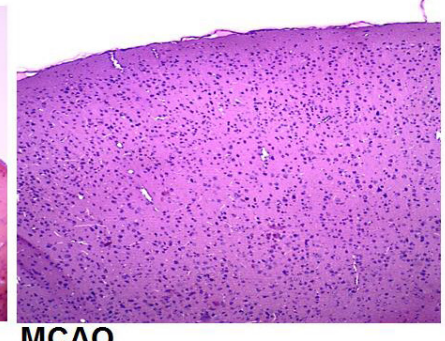

MCAO

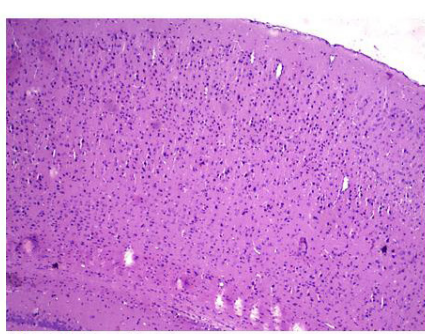

MCAO+Cell

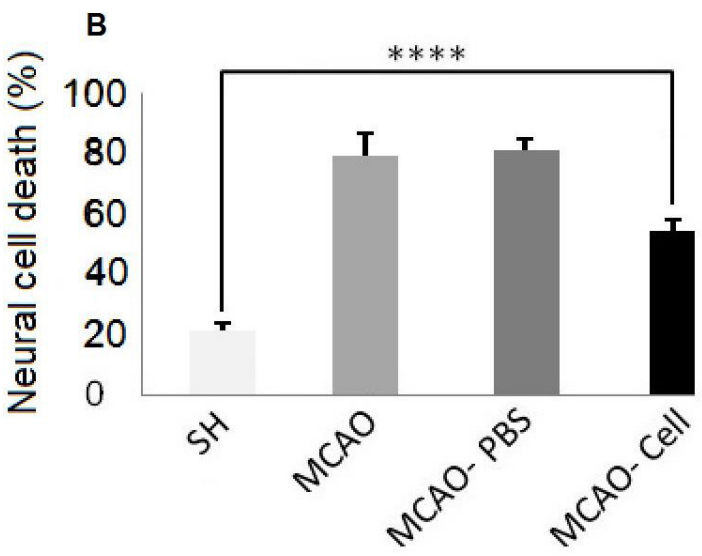

Fig. 2 Effects of hESC-NPCs ${ }^{\text {GFP }}$ on percentage of neural death of ischemic region in rat. (A) H\&E staining in different groups (100X). (B) Comparing the percentage of neural death in different groups $* * * *<0.0001$ compared to Sh group; Sh: sham operated group; MCA0: Ischemia induction group, MCAO+PBS: Ischemia induction group with ICV injection of PBS; MCAO+Cell: Ischemia induction group with ICV injection of $h E S C-N P C{ }^{G F P}$.

\section{Discussion}

In this study, we evaluated the effects of h-ESC-NPC on brain impairments induced by ischemia reperfusion. For this purpose, the neurological score, histopathological changes, and the miRNAs-120 level of ischemic area and peripheral blood were investigated in each group. The MCAO was applied by a similar method in all rats and the formation of ischemic tissue was confirmed by $\mathrm{H} \& \mathrm{E}$ staining. The MCAO+Cell group significantly had better sensory-motor function. As the previous studies showed, transplantation of ESC-NPCs in brain ischemic lesion could promotes functional recovery after ischemia reperfusion via migration proliferation, and differentiation in the ischemic region. Human ESC-NPCs proliferate and differentiate to neurons and glia cells in one step. Administration of ESC-NPCs was approved to reduce the volume of ischemic region via induction of neurogenesis and angiogenesis. ${ }^{8}$ On day 7, after cell injection, the rats were perfused and their brains were extracted for more studies. The H\&E staining results confirmed the reduced percentage of neural death in ischemic region. The migration, proliferation and differentiation of h-ESC-NPCs in the ischemic lesion has been confirmed in several studies. ${ }^{28-31}$

On the other hand, this amount of transplanted cells cannot be differentiated to replace the damaged tissue in the ischemic region. So, they might apply their alterations via other mechanisms. The transplanted cells secrete different trophic factors including cytokines, chemokines, and extracellular proteins to the surrounding environments which act as antiapoptotic factor, immunomodulators, angiogenesis factors, and antioxidant molecules. These progenitor cells are capable to endogenous neurotropic factors such as brain-derived neurotrophic factor (BDNF), stromal cell-derived factor 1 (SDF1), vascular endothelial growth factor (VEGF), nerve growth factor (NGF), etc. All these factors play important and beneficial role to repair the ischemic impairments following stem cell transplantation. The analysis of neural stem cells' secretome showed that they secrete different growth factors, 


\section{A: miR-210-Blood}

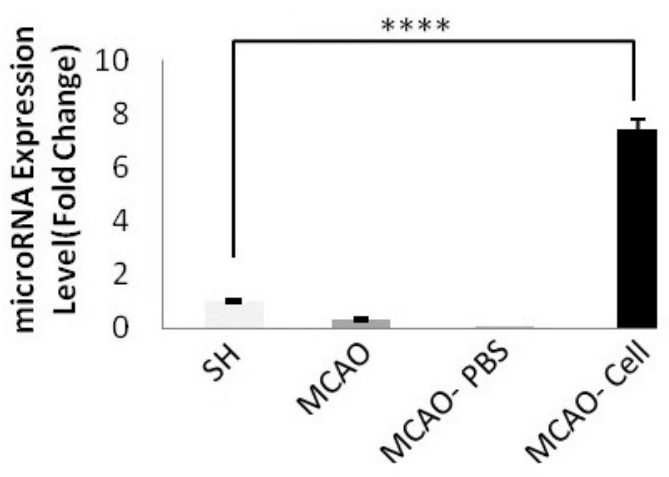

\section{C: $\operatorname{miR}-210$}
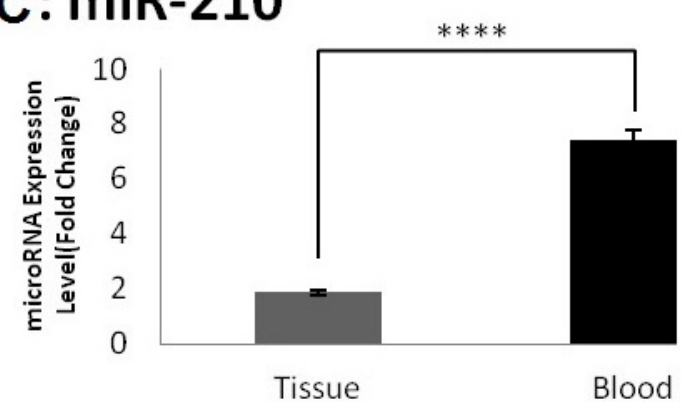

\section{B: miR-210-Tissue}
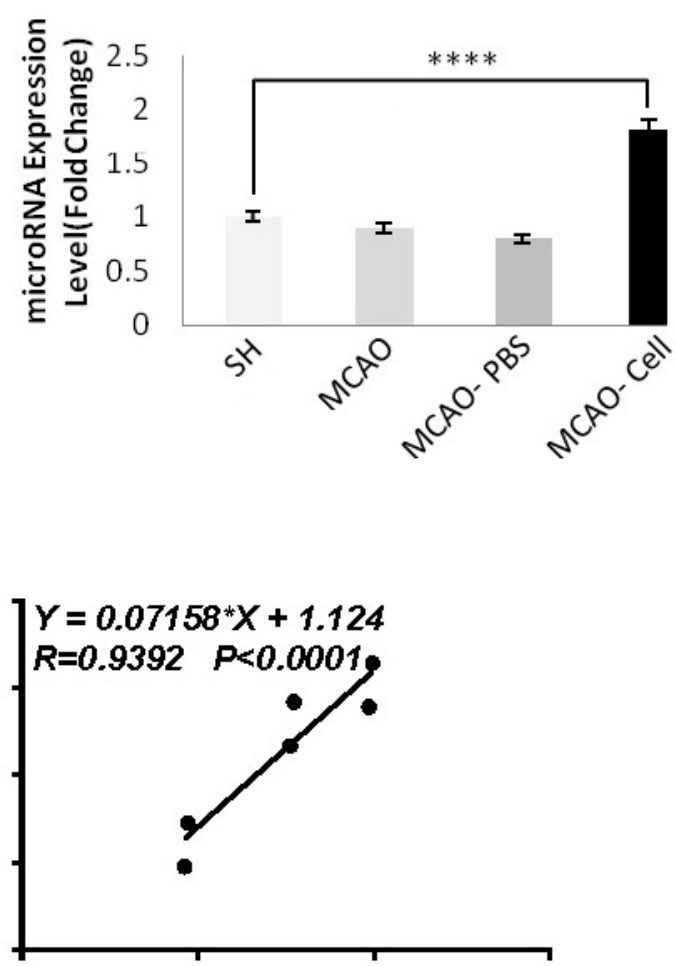

Fig. 3 Real-time PCR quantification of target gene expression in the rats' blood of all groups at 48 hours after ischemia. (A) miRNA-210 Expression in brain samples, (B) miRNA-210 Expression in blood samples. The figure shows that miRNA-210 upregulated in blood and tissue in MCAO+Cell group compared to MCAO+PBS group 48h after ischemia. ${ }^{* * * *} p<0.0001$. Data are expressed as mean \pm SEM. All reactions were performed in triplicate. (C) Correlation between rats' Blood/Tissue- $48 \mathrm{H}$ after cell injection.

e.g., VEGF, glial cell-derived neurotrophic factor (GDNF), triiodothyronine, NGF, and fibroblast growth factor 8 (FGF8), etc. $^{28,32-36}$ In our previous studies, the regulation of upstream genes (NLRP1, NLRP3, ASC, and caspase-1) and downstream genes (TNF- $\alpha$, IL-1 $\beta$, IL-18, and IL-6) of inflammasome complex has been investigated. These studies demonstrated that transplanted stem cells from different sources from human can reduce the inflammation by inhibit the formation and also action of inflammasome complexes and consequently prevent the secondary injury in nervous system. ${ }^{37-39}$

Brain and blood miRNAs helped the transplanted cells to progress the regeneration process. miRNAs keep the neural cells survived in hypoxic environment by regulating different pathway that activates in this critical condition. miRNAs has been shown to significantly enhance the survival and neuroprotective efficacy of mesenchymal stem cells for treatment of intracerebral hemorrhage model. ${ }^{40}$ The previous studies have shown that in the hypoxic events, up- or downregulation of miRNAs, induce the expression of different proteins that control the inflammation and ionic hemostasis ${ }^{41,42}$ and also has been shown that inflammation may disturb the neural regeneration. ${ }^{43}$

Therefore, to realize the effect of transplanted cells on fold change of miRNAs following ischemia, we assessed the changes of miRNA-210. The studies showed that these miRNAs might be upregulated following ischemic attack to survive affected cells. ${ }^{44}$ Our findings showed that miRNA-21 upregulated in blood and brain $48 \mathrm{~h}$ after MCAO. The most upregulation of all miRNAs was observed in $\mathrm{MCAO}+$ Cell group compared with $\mathrm{MCAO}+\mathrm{PBS}, \mathrm{MCAO}$, and sham groups. Upregulation of miRNAs was intensively seen in the blood after $48 \mathrm{~h}$ of ischemia. ${ }^{15,44}, 45$ We demonstrated that upregulation of these miRNAs is strengthened by stem cell injection, in other words, stem cells collaborate with miRNA to promote regeneration of neural cells in the ischemic region. As a detectable biomarker in blood, this factor can be used as a predictor of regeneration and prognosis of disease.

We can conclude that miRNAs are a part of regenerative process in coordination with stem cells. Hence, measuring the miRNAs in blood sample of patients with stroke can show the prognosis of patient as a biomarker of regeneration.

\section{Conclusion}

ICV injection of h-ESC-NPCs in MCAO model could improve the sensory-motor condition by decreasing the neural death and promotion of miRNA-210 in ischemic region and blood samples.

\section{Acknowledgment}

This study was registered in Department of Research, School of Advanced Technologies in Medicine, Tehran University of Medical Science with register number of 95-02-87-32255. 


\section{References}

1. Romano JG, Sacco RL. Decade in review-stroke: progress in acute ischaemic stroke treatment and prevention. Nat Rev Neurol. 2015;11(11):619-21.

2. Bagheri A, Talei S, Hassanzadeh N, Mokhtari T, Akbari M, Malek F, et al. The neuroprotective effects of flaxseed oil supplementation on functional motor recovery in a model of ischemic brain stroke: Upregulation of BDNF and GDNF. Acta Med Iran. 2017:785-92.

3. Cheng Y, Zhang J, Deng L, Johnson NR, Yu X, Zhang N, et al. Intravenously delivered neural stem cells migrate into ischemic brain, differentiate and improve functional recovery after transient ischemic stroke in adult rats. Int J Clin Exp Pathol. 2015;8(3):2928-36.

4. Alizamir T, Akbari M, Mokhtari T, Hassanzadeh G. Associated functional motor recovery induced by Intracerebroventricular (ICV) microinjection of Wharton's jelly mesenchymal stem cells following brain ischemia/ reperfusion injury in rat: Decreased dark neurons and Bax gene expression in the cerebral corte. J Contemp Med Sci. 2017;3(12).

5. Mehrannia K, Mokhtari T, Mogehi SMHN, Akbari M, Bazzaz JT, Mahakizeh $S$, et al. Intracerebroventricular injection of Wharton's jelly mesenchymal stem cells attenuates brain damage in rat model of hypoxia: optimization of vascular endothelial growth factor and downregulation of inflammatory factors. J Contemp Med Sci. 2018;4(3)

6. Hao L, Zou Z, Tian H, Zhang Y, Zhou H, Liu L. Stem cell-based therapies for ischemic stroke. Biomed Res Int. 2014;2014:468748.

7. Takagi Y, Nishimura M, Morizane A, Takahashi J, Nozaki K, Hayashi J, et al. Survival and differentiation of neural progenitor cells derived from embryonic stem cells and transplanted into ischemic brain. J Neurosurg. 2005;103(2):304-10.

8. Baker EW, Kinder HA, West FD. Neural stem cell therapy for stroke: A multimechanistic approach to restoring neurological function. Brain Behav. 2019;9(3):e01214.

9. Daadi MM, Maag AL, Steinberg GK. Adherent self-renewable human embryonic stem cell-derived neural stem cell line: Functional engraftment in experimental stroke model. PLoS One. 2008;3(2):e1644

10. Chang DJ, Oh SH, Lee N, Choi C, Jeon I, Kim HS, et al. Contralaterally transplanted human embryonic stem cell-derived neural precursor cells (ENStem-A) migrate and improve brain functions in stroke-damaged rats. Exp Mol Med. 2013;45:e53.

11. Yang ZB, Li TB, Zhang Z, Ren KD, Zheng ZF, Peng J, et al. The diagnostic value of circulating brain-specific microRNAs for ischemic stroke. Intern Med. 2016;55(10):1279-86.

12. Li M, Zhang J. Circulating microRNAs: Potential and emerging biomarkers for diagnosis of cardiovascular and cerebrovascular diseases. Biomed Res Int. 2015;2015:730535.

13. Bhalala OG, Srikanth M, Kessler JA. The emerging roles of microRNAs in CNS injuries. Nat Rev Neurol. 2013;9(6):328-39.

14. Wang C, Ji B, Cheng B, Chen J, Bai B. Neuroprotection of microRNA in neurological disorders (Review). Biomed Rep. 2014;2(5):611-9.

15. Wang Y, Wang Y, Yang GY. MicroRNAs in cerebral ischemia. Stroke Res Treat. 2013;2013:276540

16. Martinez B, Peplow PV. Blood microRNAs as potential diagnostic and prognostic markers in cerebral ischemic injury. Neural Regen Res. 2016;11(9):1375-8.

17. Qiu J, Zhou XY, Zhou XG, Cheng R, Liu HY, Li Y. Neuroprotective effects of microRNA-210 on hypoxic-ischemic encephalopathy. Biomed Res Int 2013;2013:350419.

18. Huang X, Le QT, Giaccia AJ. MiR-210--micromanager of the hypoxia pathway. Trends Mol Med. 2010;16(5):230-7.

19. Meng ZY, Kang HL, Duan W, Zheng J, Li QN, Zhou ZJ. MicroRNA-210 Promotes accumulation of neural precursor cells around ischemic foci after cerebral ischemia by regulating the SOCS1-STAT3-VEGF-C pathway. J Am Heart Assoc. 2018;7(5).

20. Chan YC, Banerjee J, Choi SY, Sen CK. miR-210: The master hypoxamir Microcirculation. 2012;19(3):215-23.

21. Kim HW, Haider HK, Jiang S, Ashraf M. Ischemic preconditioning augments survival of stem cells via miR-210 expression by targeting caspase-8associated protein 2. J Biol Chem. 2009;284(48):33161-8.

22. Zeng $L$, He $X$, Wang $Y$, Tang $Y$, Z Zheng $C$, Cai H, et al. MicroRNA-210 overexpression induces angiogenesis and neurogenesis in the normal adult mouse brain. Gene Ther. 2014;21(1):37-43.

23. Zendedel A, Habib P, Dang J, Lammerding L, Hoffmann S, Beyer C, et al. Omega-3 polyunsaturated fatty acids ameliorate neuroinflammation and mitigate ischemic stroke damage through interactions with astrocytes and microglia. J Neuroimmunol. 2015:278:200-11.

24. Mokhtari T, Akbari M, Malek F, Kashani IR, Rastegar T, Noorbakhsh F, et al. Improvement of memory and learning by intracerebroventricular microinjection of $\mathrm{T} 3$ in rat model of ischemic brain stroke mediated by upregulation of BDNF and GDNF in CA1 hippocampal region. Daru. 2017;25(1):4.

25. Nemati S, Hatami M, Kiani S, Hemmesi K, Gourabi H, Masoudi N, et al. Long-term self-renewable feeder-free human induced pluripotent stem cell-derived neural progenitors. Stem Cells Dev. 2011;20(3):503-14.

26. Chen J, Hu R, Liao H, Zhang Y, Lei R, Zhang Z, et al. A non-ionotropic activity of NMDA receptors contributes to glycine-induced neuroprotection in cerebral ischemia-reperfusion injury. Sci Rep. 2017;7(1):3575.

27. Atlasi MA, Naderian H, Noureddini M, Fakharian E, Azami A. Morphology of rat hippocampal CA1 neurons following modified two and four-vessels global ischemia models. Arch Trauma Res. 2013;2(3):124

28. Takahashi K, Yasuhara T, Shingo T, Muraoka K, Kameda M, Takeuchi A, et al. Embryonic neural stem cells transplanted in middle cerebral artery occlusion model of rats demonstrated potent therapeutic effects, compared to adult neural stem cells. Brain Res. 2008;1234:172-82.

29. Daadi MM, Hu S, Klausner J, Li Z, Sofilos M, Sun G, et al. Imaging neural stem cell graft-induced structural repair in stroke. Cell Transplant. 2013;22(5): 881-92.

30. Beyer F, Samper Agrelo I, Kury P. Do neural stem cells have a choice? Heterogenic outcome of cell fate acquisition in different injury models. Int J Mol Sci. 2019;20(2)

31. Ouyang Q, Li F, Xie Y, Han J, Zhang Z, Feng Z, et al. Meta-analysis of the safety and efficacy of stem cell therapies for ischemic stroke in preclinical and clinical studies. Stem Cells Dev. 2019;28(8):497-514.

32. Barzegar M, Kaur G, Gavins FNE, Wang Y, Boyer CJ, Alexander JS. Potential therapeutic roles of stem cells in ischemia-reperfusion injury. Stem Cell Res. 2019;37:101421.

33. Bliss T, Guzman R, Daadi M, Steinberg GK. Cell transplantation therapy for stroke. Stroke. 2007;38(2 Suppl):817-26.

34. Liao LY, Lau BW, Sanchez-Vidana DI, Gao Q. Exogenous neural stem cell transplantation for cerebral ischemia. Neural Regen Res. 2019;14(7): 1129-37

35. Willis CM, Nicaise AM, Peruzzotti-Jametti L, Pluchino S. The neural stem cel secretome and its role in brain repair. Brain Res. 2020;1729:146615.

36. Baraniak PR, McDevitt TC. Stem cell paracrine actions and tissue regeneration. Regen Med. 2010;5(1):121-43.

37. Mousavi M, Hedayatpour A, Mortezaee K, Mohamadi Y, Abolhassani F, Hassanzadeh G. Schwann cell transplantation exerts neuroprotective roles in rat model of spinal cord injury by combating inflammasome activation and improving motor recovery and remyelination. Metab Brain Dis. 2019;34(4):1117-30.

38. Mahdavipour M, Hassanzadeh G, Seifali E, Mortezaee K, Aligholi H, Shekari $F$, et al. Effects of neural stem cell-derived extracellular vesicles on neuronal protection and functional recovery in the rat model of middle cerebral artery occlusion. Cell Biochem Funct. 2019

39. Ijaz S, Mohammed I, Gholaminejhad M, Mokhtari T, Akbari M, Hassanzadeh G. Modulating pro-inflammatory cytokines, tissue damage magnitude, and motor deficit in spinal cord injury with subventricular zone-derived extracellular vesicles. J Mol Neurosci. 2020;70(3):458-66.

40. Zhang H, Wang Y, Lv Q, Gao J, Hu L, He Z. MicroRNA-21 overexpression promotes the neuroprotective efficacy of mesenchymal stem cells for treatment of intracerebral hemorrhage. Front Neurol. 2018;9:931-.

41. Slota JA, Booth SA. MicroRNAs in neuroinflammation: Implications in disease pathogenesis, biomarker discovery and therapeutic applications. Noncoding RNA. 2019;5(2).

42. RoitbakT. MicroRNAs and regeneration in animal models of CNS disorders. Neurochem Res. 2019.

43. Obora K, Onodera Y, Takehara T, Frampton J, Hasei J, Ozaki T, et al. Inflammation-induced miRNA-155 inhibits self-renewal of neural stem cells via suppression of CCAAT/enhancer binding protein $\beta$ (C/EBP $\beta$ ) expression. Scientific reports. 2017;7:43604

44. Xu W, Gao L, Zheng J, Li T, Shao A, Reis C, et al. The roles of microRNAs in stroke: Possible therapeutic targets. Cell Transplant. 2018;27(12):1778-88.

45. Jeyaseelan K, Lim KY, Armugam A. MicroRNA expression in the blood and brain of rats subjected to transient focal ischemia by middle cerebral artery occlusion. Stroke. 2008;39(3):959-66.

This work is licensed under a Creative Commons Attribution-NonCommercial 3.0 Unported License which allows users to read, copy, distribute and make derivative works for non-commercial purposes from the material, as long as the author of the original work is cited properly. 